\title{
Kerr nonlinear switching in silicon fibre-based microcylindrical resonators
}

\author{
F. H. Suhailin, ${ }^{1,2}$ N. Vukovic, ${ }^{1}$ P. Mehta, ${ }^{1}$ N. Healy, ${ }^{1}$ and A. C. Peacock ${ }^{1}$ \\ ${ }^{1}$ Optoelectronics Research Centre, University of Southampton, SO17 1BJ, United Kingdom \\ ${ }^{2}$ Physics Department, Faculty of Science and Technology, University Malaysia Terengganu, 21300 Kuala Terengganu, Malaysia \\ acp@orc.soton.ac.uk
}

\begin{abstract}
We investigate the Kerr nonlinearity in a a-Si:H microcylindrical resonator fabricated from the silicon fibre platform. The large resonant wavelength shift observed for pulsed excitation is used to demonstrate ultrafast all-optical switching.

OCIS codes: (140.4780) Optical Resonator; (140.3948) Microcavity Devices; (190.3270) Kerr Effect; (160.6000) Semiconductor Materials.
\end{abstract}

\section{Introduction}

Whispering gallery mode (WGM) microresonators with ultra-high quality factors $(Q)$ and small mode volumes are ideal platforms for the examination and exploitation of optical functionalities such as lasing, cavity quantum electrodynamics, and nonlinear optics. To date, a number of fibre-based WGM resonators have been demonstrated in various geometrical forms, including cylinders, bottles, and microspheres. Much of the work in this area has focused on resonators fabricated from glasses (e.g., silica and the chalcogenides), however, following the emergence of the new class of semiconductor fibres, more recently it has been extended to materials such as silicon and germanium [1, 2]. These semiconductor fibre-derived resonators offer advantageous properties such as extended optical transmission windows, high refractive indices for tight mode confinement, and large nonlinear coefficients.

In this paper we investigate the large Kerr nonlinearity in a hydrogenated amorphous silicon (a-Si:H) microcylindrical resonator fabricated from a silicon optical fibre. Although planar-based silicon resonators have been used to demonstrate all-optical control via relatively slow thermal or free-carrier induced nonlinear processes [3], they have yet to exploit the ultrafast nature of the Kerr effect. By comparing the resonant wavelength shifts observed when pumping with picosecond pulses versus those for a continuous wave (CW) source, we can deconvolve the Kerr and thermal contributions. Exploiting the large Kerr shift we show that picosecond all-optical switching of a CW probe is possible.

\section{Fabrication and Characterization}

The silicon microresonator was fabricated by etching the silica cladding away from a $5.9 \mu \mathrm{m}$ diameter a-Si:H core fibre fabricated using the high pressure deposition technique [1]. The fibre had a linear loss of $\sim 1.6 \mathrm{~dB} / \mathrm{cm}$ and a large Kerr nonlinear index of $\mathrm{n}_{2} \sim 1.8 \times 10^{-13} \mathrm{~cm}^{2} / \mathrm{W}$ associated with the a-Si:H material (two times larger than c-Si), as determined via transmission measurements at $1.54 \mu \mathrm{m}$ [4]. A tapered single mode fibre was used to probe the resonator's optical properties over an extended telecoms band. The experimental setup which uses just a single scanning CW laser is shown via the top (blue input and output) trace in Fig. 1(a). The transmission spectrum, as measured via an optical component tester (OCT, Yenista CT 400), is plotted in Fig. 1(b), showing two sharp resonances at $\lambda_{r 1} \sim 1533.63 \mathrm{~nm}$ and $\lambda_{r 2} \sim 1578.27 \mathrm{~nm}$. The resonances have extinction ratios of $13 \mathrm{~dB}$ and $9.5 \mathrm{~dB}$, with loaded $Q$ factors of $Q_{l, 1} \sim 1.2 \times 10^{4}$ and $Q_{l, 2} \sim 1.1 \times 10^{4}$, for $\lambda_{r 1}$ and $\lambda_{r 2}$ respectively.

(a) CWTLS

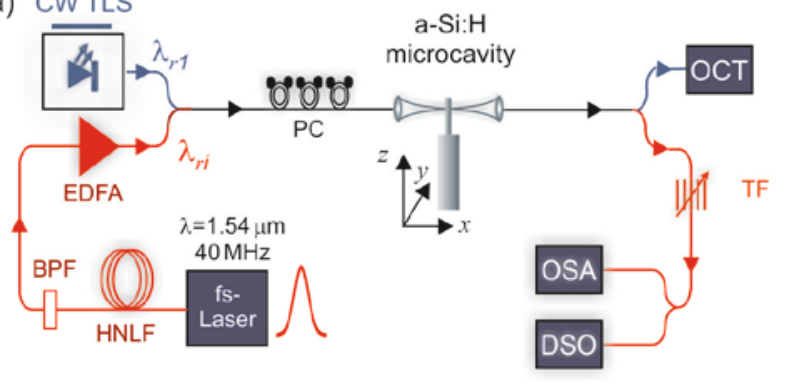

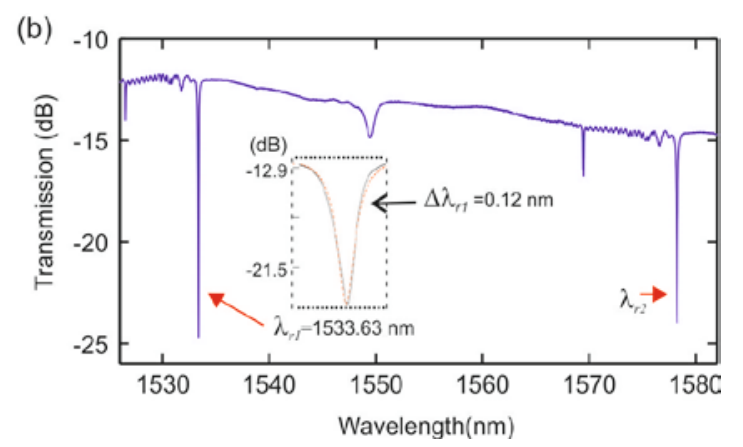

Fig. 1. (a) Setup for measuring Kerr shift and demonstrating all-optical modulation. Blue traces for CW measurements, red for pulses ( $i=1,2)$, modulation experiment combines the two. Highly nonlinear fibre (HNLF), band-pass filter (BPF), fibre amplifier (EDFA), spectrum analyzer (OSA), tunable filter (TF), digital sampling oscilloscope (DSO), component tester (OCT). (b) Transmission spectrum. Inset: Lorentzian fit to determine $Q_{l, 1}$. 


\section{Results and Discussion}

To investigate the wavelength shifting due to the Kerr and thermal effects, we use both parts of the setup shown in Fig. 1(a) separately. First the tunable CW laser is used to measure the resonant shift of $\lambda_{r 1}$ as a function of power coupled into the resonator by monitoring the position of the dip on the OCT (top blue trace), as shown by the circles in Fig. 2(a). Following this, the wavelength shifting obtained via a $\sim 2$ ps pump pulse with a broadband spectrum around $\lambda_{r 1}$ (generated via propagation of a femtosecond pulse through a nonlinear fibre and filter, bottom red trace), was measured. To ensure that the wavelength shift was recorded at the time of the pump pulse, the output of the taper was monitored via a combination of a narrow-band tunable filter (TF) and digital sampling oscilloscope (DSO). Here scope was used to monitor the amplitude of the output pulses as a function of filter wavelength, as it is tuned over the resonance, so that the position of the resonance minimum could then be determined by the observation of the maximum reduction in the transmitted pulses (i.e., when most of the light is coupled into the resonator). The corresponding large red-shifts associated with the low average coupled, but high peak power pulses are shown as the crosses in Fig. 2(a). As the small CW shift is entirely due to the thermal effects, we attribute the order of magnitude larger shifts for the pulses principally to the high Kerr nonlinearity of the a-Si:H material.
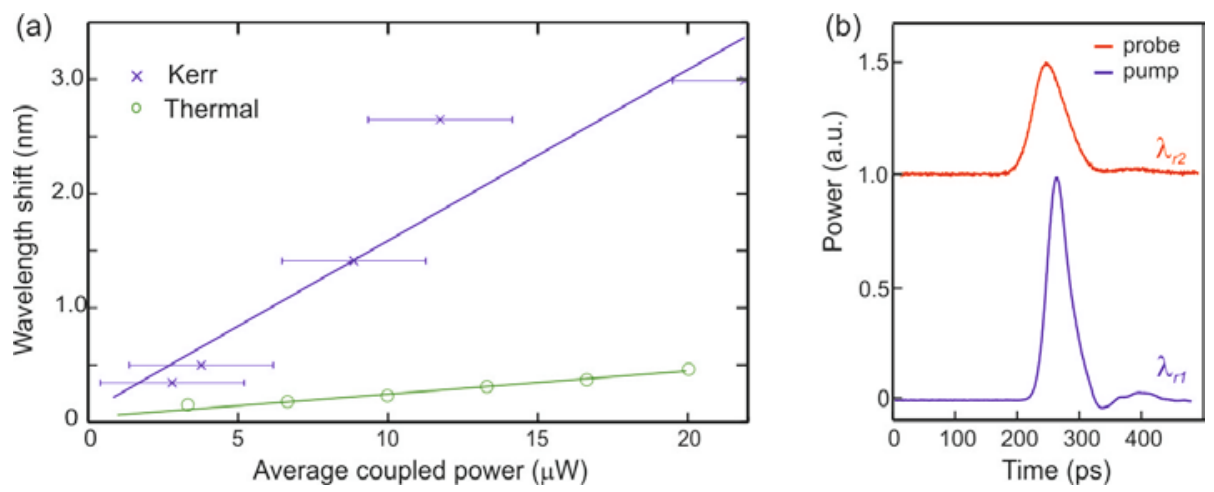

Fig. 2. (a) Wavelength shifting as functions of average power for Kerr (cross) and thermal (circle) shifts; error bars due to the uncertainty in the power coupled into the resonances in the pulsed regime. (b) All-optical switching of CW probe due to pumping with a high power, short pulse.

Finally, to exploit the Kerr effect for all optical switching, the two lasers (CW and picosecond pulsed) in Fig. 1(a) were tuned to WGM resonances $\lambda_{r 1}$ (probe, blue trace in) and $\lambda_{r 2}$ (pump, red trace in, different BPF), respectively, and launched into the tapered fibre. Here the pump is used to shift the CW probe into and out of the resonance. The average power of the pump was $11 \mu \mathrm{W}$, which corresponds to peak power of $<200 \mathrm{~mW}$. In this measurement both outputs follow the red trace and the TF was used to isolate the probe, which was monitored using the $30 \mathrm{GHz}$ DSO. Fig. 2(b) illustrates the observed ultrafast all-optical switching that occurs when the Kerr induced index change is used to shift the resonances. To record the pulses at the output, a filter is applied to the bandwidth limited detection system (which cannot accurately resolve the picosecond response times), and this results in a stretching of the time axis. However, owing to the near instantaneous response of the Kerr effect we expect the onoff switching time to be on the order of the cavity lifetime $\tau=Q / \omega \sim 10 \mathrm{ps}$, an order of magnitude faster than previous reports for free-carrier induced switching [3]. The resulting picosecond bright pulse exhibits a modulation extinction ratio of $\sim 3 \mathrm{~dB}$, which is not unreasonable for a silicon modulator.

In conclusion, the first demonstration of the Kerr effect in a-Si:H fibre based microresonators is presented. We show that the power threshold needed to induce Kerr nonlinearity is only a few $\mu \mathrm{W}$ in a picosecond pulse excitation regime and that this can be exploited for ultra-low power, ultrafast switching. Such compact, low power, high speed devices are expected to have wide ranging applications, not only in signal processing, but also in fundamental scientific investigations.

The authors thank the Department of Chemistry and Materials Research Institute of the Pennsylvania State University and Todd D. Day for the fabrication of the silicon fibre.

\section{References}

[1] N. Vukovic, N. Healy, P. Horak, J.R. Sparks, P. J. A. Sazio, J. V. Badding, A. C. Peacock,"Ultra-smooth microcylindrical resonators fabricated from the silicon optical fiber platform," Appl. Phys. Lett. 99, 031117 (2011).

[2] P. Wang, T. Lee, M. Ding, A. Dhar, T. Hawkins, P. Foy, Y. Semenova, Q. Wu, J. Sahu, G. Farrell, J. Ballato, G. Brambilla, "Germanium microsphere high-Q-resonator," Opt. Lett. 37, 728 (2012).

[3] Q. Xu and M. Lipson, "Carrier-induced optical bistability in silicon ring resonators," Opt. Lett. 31, 341 (2006).

[4] P. Mehta, N. Healy, N. F. Baril, P.J.A. Sazio, J. V. Badding, A. C. Peacock, "Nonlinear transmission properties of hydrogenated amorphous silicon core optical fibers," Opt. Express 16, 16826-16831 (2010). 